\title{
Onlay Rib Bone Graft in Elevation of Reconstructed Auricle: 17 Years of Experience
}

\author{
Taehoon Kim, Jihyeon Han, Yoonho Lee \\ Department of Plastic and Reconstructive Surgery, Seoul National University College of Medicine, Seoul, Korea
}

Background A cartilage wedge block and covering flap are standard procedures for firm elevation of the ear in microtia correction. However, using costal cartilage for elevation of the reconstructed auricle can be insufficient, and the fixed cartilage wedge block may be absorbed or may slip out. Furthermore, elevating covering flaps is time-consuming and uses up fascia, a potential source of reconstruction material. Therefore, we propose an innovative method using autologous onlay rib bone graft for auricular elevation of microtia.

Methods From February 1995 to August 2012, 77 patients received a first stage operation with a rib cartilage framework graft. In the second stage operation, a small full thickness of rib bone was harvested through the previous donor scar. The bihalved rib bone was inserted into the subperiosteal pocket beneath the cartilage framework.

Results The follow-up time ranged from 1 month to 17 years, with a mean of 3 years. All of the patients sustained the elevation of their ears very well during the follow-up period. Donor site problems, except for hypertrophic scars, were not observed. Surgery-related complications, specifically skin necrosis, infection, or hematoma, occurred in 4 cases.

Conclusions Onlay rib bone graft used to elevate the reconstructed auricle is a more anatomically appropriate material than cartilage, due to the bone-to-bone contact between the bone graft and the temporal bone. Postoperative minor correction of the elevation degree is straightforward and the skin graft survives better. Therefore, reconstructed auricle elevation using onlay rib bone graft is a useful and valuable method.

Keywords Bone transplantation / Cartilage / Ear auricle
Correspondence: Yoonho Lee Department of Plastic and Reconstructive Surgery, Seoul National University College of Medicine, 101 Daehak-ro, Jongno-gu, Seoul 110-744, Korea

Tel: $+82-2-2072-2374$

Fax: +82-2-3675-7792

E-mail: lyh2374@snu.ac.kr

This article was presented at the 70th Congress of the Korean Society of Plastic and Reconstructive Surgeons on November 9-11, 2012 in Seoul, Korea.

No potential conflict of interest relevant to this article was reported.

\section{INTRODUCTION}

Although numerous corrective procedures are available, microtia reconstruction remains challenging for plastic surgeons. Since the introduction of Tanzer's technique using autologous costal cartilage [1-3], a variety of modifications have been developed [4-14]. Nowadays, Brent or Nagata's technique [5-13] is widely used. In both of the authors' techniques, a cartilage wedge block and covering flap (mastoid fascial flap or temporoparietal fascial flap) have been used for firm elevation of the ear in microtia correction. However, using costal cartilage for elevation of the reconstructed auricle can be insufficient, and the fixed cartilage wedge block is sometimes absorbed or slips out, resulting in unsatisfactory ear projection $[15,16]$. Furthermore, elevating covering flaps is time-consuming and uses up fascia, a potential source of reconstruction material in case of need for 
secondary repair after trauma. Additionally, a temporo-parietal fascial flap results in some degree of alopecia, which may be quite conspicuous, especially in patients with short hair. Therefore, we propose an innovative method using autologous onlay rib bone graft for auricular elevation of microtia.

\section{METHODS}

From February 1995 to August 2012, auricle elevation procedures were performed in 77 patients with microtia. All of the operations were conducted in the authors' hospital. Of the 77 microtia patients, 49 were right-sided (63.6\%), 27 were leftsided (35.1\%), and 1 was bilateral (1.3\%). Fifty-four (70.1\%) of the patients were male and $23(29.9 \%)$ were female. All of the patients underwent a two-stage operation.

\section{First stage operation: rib cartilage framework graft}

The costal cartilage of the sixth to eighth ribs on the ipsilateral side is harvested together with the anterior costal perichondrium intact. The base frame is then constructed from the sixth to seventh costal cartilage and the helix is constructed from the eighth. The antihelix, superior and inferior crus unit, and tragus are constructed from the remains of the sixth to seventh costal cartilages. A subcutaneous pocket is made, and the fabricated three-dimensional framework is inserted into it. The procedures in the first stage operation follow Nagata's method [9-12].

\section{Second stage operation: elevation of the reconstructed auricle}

The secondary surgery is performed 6 months after the first surgery. The skin incision line is made $5 \mathrm{~mm}$ outside of the margin of the reconstructed auricle. The implanted cartilage framework is elevated from the underlying bed. Dissection is performed in the sub-fascial plane for better skin graft survival. The rib bone is harvested through the previous donor scar site.
As in the costal cartilage harvest method, a rib bone about 4 to $6 \mathrm{~cm}$ in size is harvested with the anterior costal periosteum intact. The harvested rib bone is usually bihalved with an electronic reciprocating saw. Retrograde dissection fanning outward from the reconstructed auricle skin incision site is performed in the suprafascial plane. An additional 1 to $2 \mathrm{~cm}$ incision is made through the mastoid fascia and its underlying periosteum. A subperiosteal pocket is created beneath the costal cartilage framework by subperiosteal dissection. The bihalved rib bones are inserted into the subperiosteal pocket with the cortical bone oriented outward. For anatomical elevation of the reconstructed ear using a bone graft, accentuation of an inverted L-shape protuberance on the temporal bone is mandatory. Fixation of the rib bone graft is not necessary because the frictional force made by contact between the cancellous part of the bihalved rib bone and the temporal bone is sufficient to prevent slippage. The posterior scalp flap is advanced anteriorly and fixed with full-layer 2-0 nylon suture to reduce the area requiring skin graft (Fig. 1). A full-thickness skin graft obtained from the groin area is grafted to the remaining posterior raw surface of the elevated auricle and the mastoid and temporal surfaces. Tie-over dressing is performed over the skin graft site. The tie-over sutures are removed after 5 days. The patient is discharged with an ear protection cap at postoperative day 7 . All sutures are completely removed after the second week at the outpatient clinic.

\section{RESULTS}

In this series, the follow-up period in 77 patients ranged from 1 month to 17 years, with a mean of 3 years. The average duration of the operation was 4 hours. Although there were slight decreases in elevation degree as time passed, ear elevation was well sustained during the follow-up period for all of the patients (Figs. 2-4). Patients that used an ear protection cap for 1 month postoperatively did not complain of compression symptoms

\section{Fig. 1. Intraoperative views of the rib bone graft}

(A) After an additional incision is made through the mastoid fascia and the underlying periosteum, a subperiosteal pocket is created beneath the costal cartilage framework by subperiosteal dissection. (B) The bihalved rib bones are inserted into the subperiosteal pocket with the cortical bone oriented outward. (C) The ear elevated by the rib bone graft can be seen. The posterior scalp flap is advanced anteriorly and fixed with full-layer 2-0 nylon suture to reduce areas requiring skin graft.

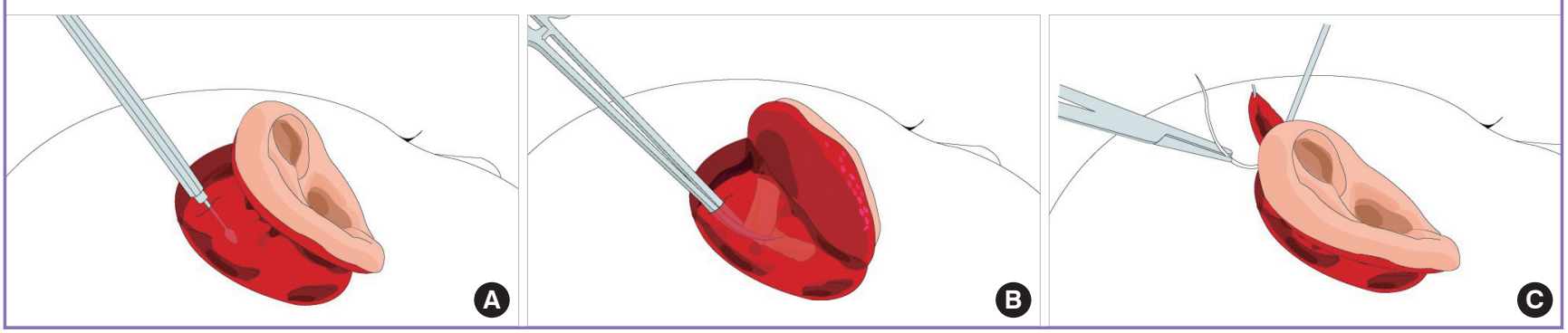




\section{Fig. 2. An 11-year-old patient with right-sided microtia}

(A) Preoperative view. (B) The appearance of the patient 1 month after ear elevation. (C) One year postoperative anterior view. The reconstructed ear is at a similar horizontal level to the normal ear, producing an acceptable appearance with glasses. (D) One year postoperative oblique view. The degree of elevation is slightly lower than in the postoperative 1 month photo.
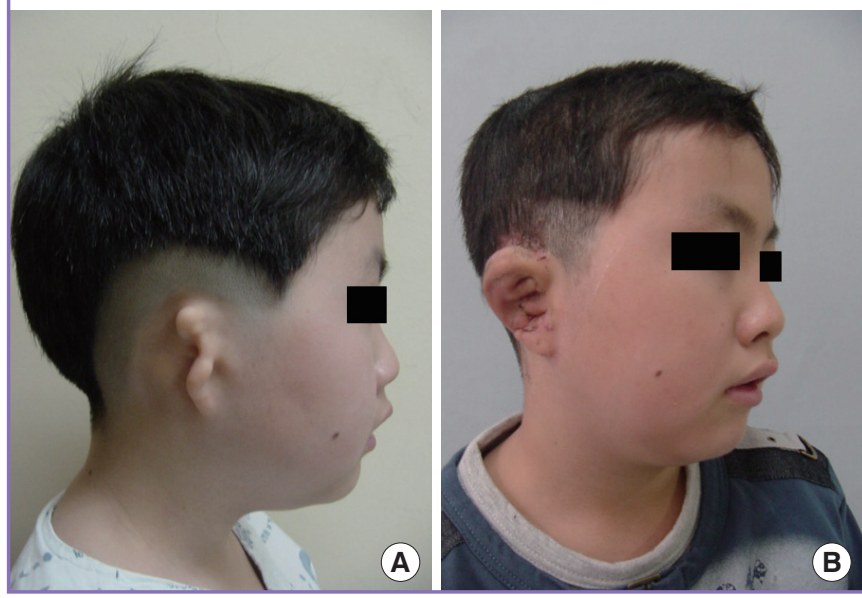

Fig. 3. A 12-year-old patient with left-sided microtia

(A) Normal side. (B) The appearance of the patient 2 years and 2 months after ear elevation. The symmetry of both ears has been sustained very well.
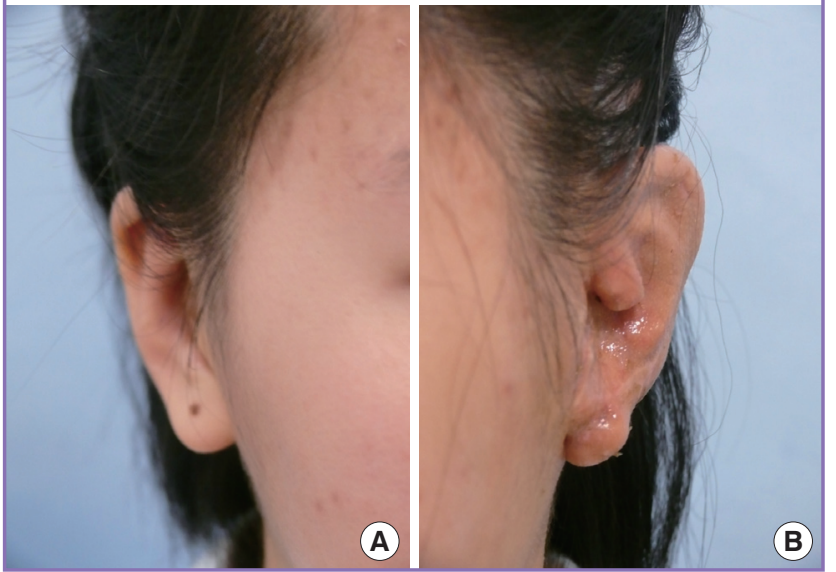

with the use of a pillow. Problems related to rib bone harvesting, such as pneumothorax, hemothorax, or delayed wound healing, were not observed. However, hypertrophic scars on the chest were seen in 5 patients. They were successfully treated with multiple intralesional triamcinolone injection. Complications in the recipient site included 1 case of hematoma, 2 cases of skin necrosis that were successfully treated with temporal fascial flap covers, and 1 case of infection that healed after drainage and antibiotic treatment.

\section{DISCUSSION}

In patients with microtia, ear elevation for the constructed auricle is a very difficult and complicated operation. In the past,
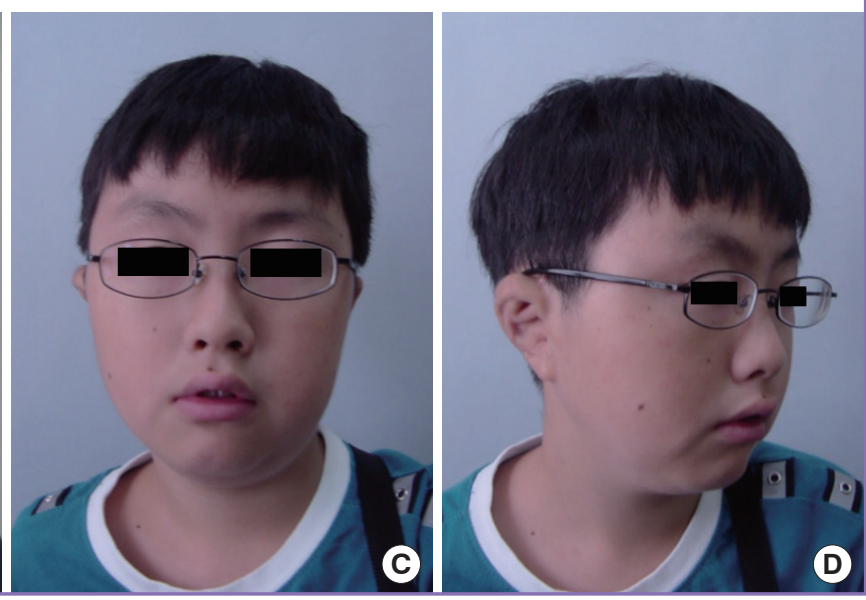

Fig. 4. Long-term follow-up appearance after 17 years

(A) This patient underwent ear elevation at 6 years of age. Although bony resorption over time is inevitable to some degree, the elevation has been maintained for 17 years. (B) Normal side.
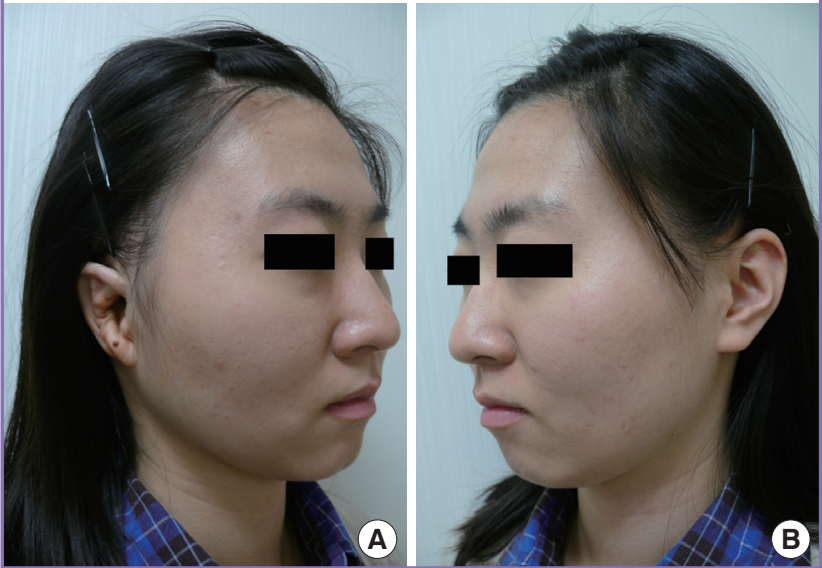

Steffensen et al. [17,18] used local flaps and skin grafts [19]. These are relatively easy techniques and have low complication rates. However, it is difficult to maintain the correct projection of the constructed auricle using these methods, which employ only soft tissue. In 1959, a two-stage ear elevation procedure using autologous costal cartilage was reported by Tanzer [1], followed by development of a variety of modifications [4-14].

These days, Brent or Nagata's technique is widely used. Brent reported a four-stage, step-by-step reconstruction method including insertion of an autologous cartilaginous framework into a skin pocket, transposition of the lobule, elevation of the ear with the remnant of the costal cartilage block and mastoid fascial flap, and construction of the tragus [5-8]. Nagata [9-13] developed a two-stage reconstruction method. During the first 


\section{Fig. 5. Postoperative CT images}

Postoperative computed tomography (CT) images of a 25-year-old man 8 months after left ear elevation. (A) Axial images showing the sections of the inverted L-shape rib bone graft. (B) Three-dimensional CT images of the normal side, in which an inverted L-shape protuberance in the temporal bone immediately posterior to the external auditory canal can be identified as a normal anatomical structure. (C) Three-dimensional CT images of the microtia side. A well-taken inverted L-shaped bihalved rib bone graft can be observed.
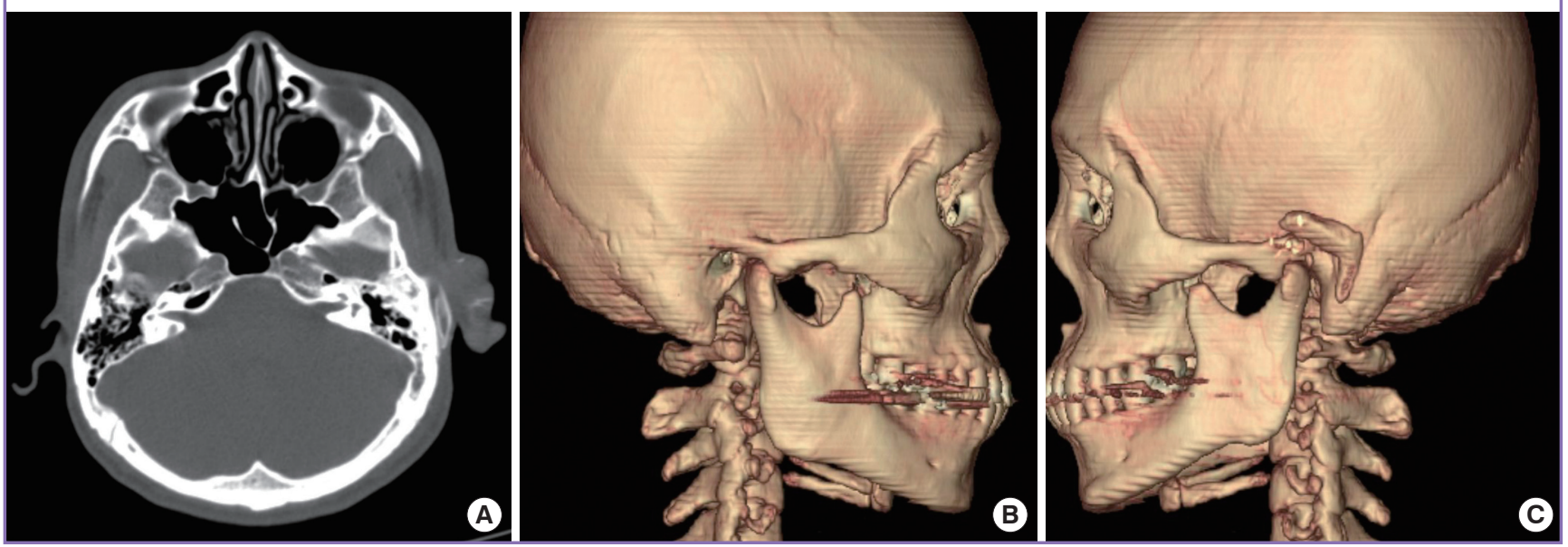

stage, a complete cartilage framework including the tragus is inserted into a skin pocket, and lobule transposition is done simultaneously. During the second stage, the reconstructed ear is raised with a costal cartilage block and temporo-parietal fascial flap [9-13]. In both authors' techniques, the cartilage wedge block must be covered with a fascial flap (e.g., mastoid fascial flap or temporoparietal fascial flap) to enhance skin graft survival. Elevating covering flaps is time-consuming and uses up fascia, which can be a potential source of reconstruction material in case there is a need for secondary repair after skin necrosis or trauma. Additionally, temporoparietal fascial flap results in some degree of alopecia, which may be quite conspicuous, especially in patients with short hair. Moreover, the remaining costal cartilage block might be insufficient and the fixed cartilage wedge block may be absorbed or slip out, resulting in unsatisfactory ear projection $[15,16]$.

Some authors have recently reported that not only the costal cartilage but also artificial materials such as hydroxyapatite or porous polyethylene or titanium mesh strut could be used for elevation [20-22]. However, artificial materials lack safety and reliability, as well as published results of research on long-term outcomes.

The onlay rib bone graft that we have designed for ear elevation can solve most of the problems mentioned above. Our method produces anatomical elevation with good projection. An inverted L-shape protuberance in the temporal bone exists immediately posterior to the external auditory canal, as an anatomically normal structure. This is almost the equivalent of the eminentia conchae location, which Nagata emphasizes as the crucial point for ear elevation [13]. Bone-to-bone contact is made between the rib bone graft and the area of protuberance in the temporal bone. The rib bone graft adheres to the underlying temporal bone by the natural bone remodeling process (Fig. 5). Moreover, there is no need for laborious flap coverage, thereby saving surgical time and preserving the temporo-parietal fascia and mastoid fascia for secondary reconstruction.

Our elevation method allows postoperative minor correction of elevation degree. The rib bone graft in the subperiosteal pocket can be shifted anteriorly, changing the pivot point of elevation in an anterior direction. The rib bone graft is held in its location by frictional force alone and is movable in the early postoperative period. This way, the ear can gain more projection.

In our method, applying a skin graft is easier and the graft survives better because the ear flap is elevated along with the superficial fascia, which can act as a bed for the skin graft. We usually reserve the deep fascia so that secondary reconstruction with the mastoid fascial flap is possible. Deep dissection to the supraperiosteal layer can be done as needed for better skin graft survival.

In bone grafts, there is always the problem of bone resorption. As expected, this phenomenon was observed in our series. In Fig. 2 , the degree of elevation at 1 year postoperatively is slightly below that at 1 month postoperatively. However, the decrease in the degree of elevation is not significant. Surprisingly, our cases included a patient with an ear projection that was maintained for 17 years (Fig. 4).

In brief, the use of a rib bone graft has several advantages over a cartilage wedge block and covering flap method. Because the authors' method conserves the covering flap, it requires less time and makes secondary reconstruction possible. Furthermore, the 
use of a bone graft prevents slip-outs and reduces absorption, while permitting minor postoperative corrections in the degree of elevation. Lastly, the ear flap contains a more reliable bed for a better skin graft survival rate.

In our study, the only donor site morbidity seen was a hypertrophic scar, which was successfully treated with multiple intralesional triamcinolone injections. Complications related to rib bone harvesting, such as pneumothorax, hemothorax, and delayed wound healing, were not observed. For the majority of the cases, we harvested a short 4 to $6 \mathrm{~cm}$ rib bone that could be fully visualized without extending the incision line, and excessive dissection was not needed. Extra caution was taken when harvesting longer lengths of rib bone in patients with ribs that could not be bihalved due to insufficient bone thickness.

It is worth considering the potential instability of the rib cage after harvesting. However, in comparison to the authors of preceding studies, we used only needed amounts during rib cartilage framework graft. Because the rib cartilage was not obtained for purposes of elevation, rib cage stability was higher than when more rib cartilage had been obtained for ear elevation. Thus, it is believed that rib cage stability does not significantly decrease due to the removal of rib bone. Moreover, because only a minimal amount of 4 to $6 \mathrm{~cm}$ of rib bone is removed and only from 1 rib among the 12 rib bones, no negative effect on the stability of the rib cage will be evident. In fact, there no patients visited the hospital due to trauma of the rib cage after this operation.

\section{REFERENCES}

1. Tanzer RC. Total reconstruction of the external ear. Plast Reconstr Surg Transplant Bull 1959;23:1-15.

2. Tanzer RC. An analysis of ear reconstruction. Plast Reconstr Surg 1963;31:16-30.

3. Converse JM. Reconstructive plastic surgery. 2nd ed. Philadelphia: W. B. Saunders; 1977.

4. Fukuda O. Long-term evaluation of modified Tanzer ear reconstruction. Clin Plast Surg 1990;17:241-9.

5. Brent B. The correction of mi-rotia with autogenous cartilage grafts: I. The classic deformity? Plast Reconstr Surg 1980;66:1-12.

6. Brent B. A personal approach to total auricular construction: case study. Clin Plast Surg 1981;8:211-21.

7. Brent $B$. The versatile cartilage autograft: current trends in clinical transplantation. Clin Plast Surg 1979;6:163-80.

8. Brent B. Technical advances in ear reconstruction with autogenous rib cartilage grafts: personal experience with 1200 cases. Plast Reconstr Surg 1999;104:319-34.

9. Nagata S. A new method of total reconstruction of the auricle for microtia. Plast Reconstr Surg 1993;92:187-201.

10. Nagata $S$. Modification of the stages in total reconstruction of the auricle: Part I. Grafting the three-dimensional costal cartilage framework for lobule-type microtia. Plast Reconstr Surg 1994;93:221-30.

11. Nagata S. Modification of the stages in total reconstruction of the auricle: Part II. Grafting the three-dimensional costal cartilage framework for concha-type microtia. Plast Reconstr Surg 1994;93:231-42.

12. Nagata S. Modification of the stages in total reconstruction of the auricle: Part III. Grafting the three-dimensional costal cartilage framework for small concha-type microtia. Plast Reconstr Surg 1994;93:243-53.

13. Nagata S. Modification of the stages in total reconstruction of the auricle: Part IV. Ear elevation for the constructed auricle. Plast Reconstr Surg 1994;93:254-66.

14. Ou LF, Yan RS, Tang YW. Firm elevation of the auricle in reconstruction of microtia with a retroauricular fascial flap wrapping an autogenous cartilage wedge. Br J Plast Surg 2001;54:573-80.

15. Zhang Q, Zhang R, Xu F, et al. Auricular reconstruction for microtia: personal 6-year experience based on 350 microtia ear reconstructions in China. Plast Reconstr Surg 2009;123: 849-58.

16. Zhang $\mathrm{Q}$ Zhang R, Xu F, et al. Firm elevation of the reconstructed auricle with a retroauricular fascial flap wrapping an EH (a mixture of epoxide acrylate malelic and hydroxyapatite) composite wedge. J Plast Reconstr Aesthet Surg 2010;63:1452-8

17. Steffensen WH. Comments on total reconstruction of the ear. Plast Reconstr Surg (1946) 1952;10:186-90.

18. Steffensen WH. Comments on reconstruction of the external ear. Plast Reconstr Surg (1946) 1955;16:194-200.

19. Ogino Y, Yoshikawa Y. Plastic surgery for the congenital anomaly of the ear. Keisei Geka 1963;6:79.

20. Tai Y, Tanaka S, Fukushima J, et al. Refinements in the elevation of reconstructed auricles in microtia. Plast Reconstr Surg 2006;117:2414-23.

21. Yaremchuk MJ. Facial skeletal reconstruction using porous polyethylene implants. Plast Reconstr Surg 2003;111:181827.

22. Li D, Zhang R, Zhang Q, et al. Titanium mesh strut: a novel instrument for firm elevation of the reconstructed auricle. Aesthetic Plast Surg 2012;36:746-9. 